\title{
Bacteremia Caused by Extended-Spectrum Beta-Lactamase-Producing Enterobacteriaceae in Vientiane, Lao PDR: A 5-Year Study
}

Ko Chang, ${ }^{1}$ Sayaphet Rattanavong, ${ }^{2}$ Mayfong Mayxay, ${ }^{2,3,4}$ Valy Keoluangkhot, ${ }^{1}$ Viengmon Davong, ${ }^{2}$ Manivanh Vongsouvath, ${ }^{2}$ Manophab Luangraj, ${ }^{2}$ Andrew J. H. Simpson, ${ }^{2,3 *}$ Paul N. Newton, ${ }^{2,3,5}$ and David A. B. Dance ${ }^{2,3,5}$

${ }^{1}$ Adult Infectious Diseases Ward, Mahosot Hospital, Vientiane, Laos; ${ }^{2}$ Lao-Oxford-Mahosot Hospital-Wellcome Trust Research Unit (LOMWRU), Microbiology Laboratory, Mahosot Hospital, Vientiane, Laos; ${ }^{3}$ Centre for Tropical Medicine and Global Health, University of Oxford, Oxford, United Kingdom; ${ }^{4}$ Institute of Research and Education Development (IRED), University of Health Sciences, Vientiane, Laos; ${ }^{5}$ Faculty of Infectious and Tropical Diseases, London School of Hygiene and Tropical Medicine, London, United Kingdom

\begin{abstract}
Although there has been an increasing incidence of bacteremia caused by extended-spectrum betalactamase (ESBL)-producing Enterobacteriaceae (ESBL-E) across South East Asia, there are sparse data from the Lao PDR, where laboratory capacity for antimicrobial resistance surveillance is limited. We, therefore, retrospectively reviewed bacteremia caused by ESBL-producing Escherichia coli and Klebsiella pneumoniae between 2010 and 2014 at Mahosot Hospital, Vientiane, Lao PDR. Clinical and laboratory data relating to all episodes of ESBL-E bacteremia were reviewed over the 5-year period and compared with non-ESBL-E bacteremia. Blood cultures positive for $E$. coli or K. pneumoniae were identified retrospectively from laboratory records. Clinical and laboratory data were extracted from research databases and case notes and analyzed using STATA. Between 2010 and 2014, we identified 360 patients with E. coli $(n=249)$ or $K$. pneumoniae $(n=111)$ bacteremia, representing $34.8 \%$ of all patients with clinically significant bacteremia. Seventy-two (20\%) isolates produced ESBL; E. coli accounted for $15.3 \%(55 / 360)$ and $K$. pneumoniae for $4.7 \%(17 / 360)$, respectively. The incidence of ESBL-producing $E$. coli bacteremia rose during the study period. By multiple logistic analysis, reported antibiotic use in the previous week was significantly associated with ESBL positivity $(P<0.001$, odds ratio 3.89). Although multiresistant, most ESBL-producing E. coli and $K$. pneumoniae remained susceptible to meropenem $(65 / 65 ; 100 \%)$ and amikacin $(64 / 65 ; 98.5 \%)$. We demonstrated an alarming increase in the incidence of ESBL-E as a cause of bacteremia in Vientiane during the study period. This has implications for empiric therapy of sepsis in Laos, and ongoing surveillance is essential.
\end{abstract}

\section{INTRODUCTION}

Bloodstream infection is a major cause of morbidity and mortality, comparable with major traumatic injury, myocardial infarction, and stroke with an annual incidence of 140-160 per 100,000 population. ${ }^{1,2}$ Antimicrobial resistance (AMR) leads to increased mortality, length of hospital stay, and hospital costs associated with bloodstream infections, including both community-onset and hospital-acquired bacteremia. ${ }^{3,4}$ Gram-negative bacteria belonging to the family Enterobacteriaceae are associated with infections ranging from minor urinary tract infections to life-threatening bacteremia. Enterobacteriaceae, especially Escherichia coli and Klebsiella pneumoniae, are becoming a major threat to public health because of their ability to acquire resistance to most current antibiotics, especially third-generation cephalosporins, such as ceftriaxone, through the production of extended-spectrum beta-lactamases (ESBLs). ${ }^{5}$ The WHO has listed ESBL-producing Enterobacteriaceae (ESBL-E) as critical priority pathogens for research and development of new antibiotics. ${ }^{6}$ Extended-spectrum beta-lactamase-producing Enterobacteriaceae have emerged as important pathogens in many countries in the Asia-Pacific region, such as India and China where ESBL-producing isolates account for $77 \%$ and $48 \%$ of Enterobacteriaceae from intra-abdominal infections, respectively. ${ }^{7}$ Lao PDR is a land-locked country, surrounded by countries with high incidence rates of ESBL-E infection, ${ }^{8,9}$ although it has few microbiology laboratories and data on AMR are, therefore, scarce. Phetsouvanh et al. ${ }^{10}$ demonstrated that

*Address correspondence to Andrew J. H. Simpson, Lao-OxfordMahosot Hospital-Wellcome Trust Research Unit (LOMWRU), Microbiology Laboratory, Mahosot Hospital, Vientiane, Laos. E-mail: andrew.s@tropmedres.ac
Gram-negative bacteria accounted for $77 \%$ of communityacquired bacteremia in Lao PDR between 2000 and 2004, with E. coli and $K$. pneumoniae accounting for $12 \%$ and $4 \%$, respectively. The first ESBL-producing $E$. coli was identified in Mahosot Hospital, Vientiane, in 2004, and between 2004 and $2009,9 \%$ of $E$. coli isolated from blood were ESBL producers. ${ }^{11}$ A study of preschool children in and around Vientiane in 2011 found that $23 \%$ were colonized with ESBL-E, mainly E. coli and $K$. pneumoniae, and colonization was strongly associated with prior antibiotic use. ${ }^{12}$ Cephalosporins, particularly ceftriaxone, are widely used for empirical treatment of febrile patients in Laos, but the lack of access to diagnostic microbiology and AMR surveillance data mean that clinicians are largely unaware of the extent of the AMR problem, leading to delays in initiating appropriate treatment. Therefore, we retrospectively reviewed the proportion, clinical features, risk factors, and antibiotic susceptibility profiles of $E$. coli and $K$. pneumoniae causing bacteremia at Mahosot Hospital, Vientiane, Laos, between 2010 and 2014.

\section{MATERIALS AND METHODS}

Mahosot Hospital in Vientiane, Laos, is a central, tertiary hospital with 450 beds, which serves a population of approximately 800,000 people in the immediate catchment, but also acts as a referral center for other peripheral and provincial hospitals. It receives approximately 19,000 admissions per year (Mahosot Summary Report 2010-2014, unpublished data). The Mahosot Hospital Microbiology Laboratory provides a bacterial culture service for Mahosot Hospital and several other hospitals in Laos, supported by the Lao-OxfordMahosot Hospital-Wellcome Trust Research Unit. It receives approximately 6,500 blood culture sets annually, of which 4,600 come from inpatients at Mahosot Hospital. As part of an 
ongoing study of the causes of fever at Mahosot Hospital since 2000 (the "Ul-study"), a set of blood cultures (two aerobic bottles [Pharmaceutical Factory Number 2, Vientiane, Laos]) is sent by the responsible clinician from all patients admitted with suspected community-acquired bacteremia, subject to written informed consent. Clinical and laboratory information at the time of study admission is recorded on standard forms and databases. Blood cultures were processed manually as described, ${ }^{10}$ with significant isolates identified phenotypically (by API 20E [bioMérieux, Basingstoke, United Kingdom] in the case of Enterobacteriaceae). Significant isolates were tested for antimicrobial susceptibility by disk diffusion according to the current methods of the Clinical and Laboratory Standards Institute. Any E. coli or Klebsiella spp. resistant to cefpodoxime, ceftriaxone or cefotaxime, or ceftazidime was tested for ESBL activity by disk diffusion against ceftazidime and cefotaxime with and without clavulanic acid: an increase in zone diameter $\geq 5 \mathrm{~mm}$ for either agent in the presence of clavulanic acid was considered as positive for ESBL. ${ }^{13}$ Molecular detection methods were not used in this study.

Data collection and analysis. We retrospectively reviewed laboratory records to identify all Mahosot Hospital patients who had blood cultures positive for $E$. coli and $K$. pneumoniae between 2010 and 2014. Clinical and microbiological data (including reported antibiotic use in the previous week) were exported from the Ul-study database into Microsoft Excel (Microsoft Corporation, Redmond, WA). Missing and inconsistent data were checked from patient charts (where available) and primary laboratory sources (e.g., laboratory work books). Where the results could not be reconciled, the organisms were re-cultured from the stock frozen at $-80^{\circ} \mathrm{C}$ and retested.

Data analysis was performed using STATA v 14.2 (Stata Corporation, College Station, TX). Data were summarized using descriptive statistics such as number, percentage, median, mean, and $95 \% \mathrm{Cl}$. Categorical variables were compared using the chi-square test or Fisher's exact test. We included the statistically significant $(P<0.05)$ and relevant variables from the univariable analysis in a multiple logistic regression model using a backward stepwise approach to identify factors associated with ESBL positivity and mortality associated with ESBL positivity.

\section{RESULTS}

During the period 2010-2014, the laboratory received 18,319 blood culture sets from 15,665 patients, of whom $6.6 \%(1,032 /$ $15,665)$ grew clinically significant organisms (i.e., excluding duplicates). Escherichia coli and $K$. pneumoniae together accounted for $34.8 \%(360 / 1,032)$ of all clinically significant isolates, of which $90 \%$ were classed as community-acquired bacteremia, that is, taken within 48 hours of hospital admission. Of these, 72 (20\%) were ESBL positive and $288(80 \%)$ were ESBL negative. In the ESBL-producing group, E. coli and $K$. pneumoniae accounted for $15.3 \%$ (55/360) and 4.7\% (17/360), respectively. The proportion of ESBL-producing $E$. coli was greater than ESBL-producing $K$. pneumoniae (15.3\% versus $4.7 \%, P<0.001$ ) throughout the study period. The proportion of $E$. coli-producing ESBL increased more than 4-fold from four (7.8\%) cases in 2010 to $17(34.7 \%)$ cases in 2014 (Figure 1).

Basic clinical and epidemiological features of the patients are shown in Table 1. Of the 360 patients, 143 (40\%) were male and the overall median interquartile range (IQR) age was 57
(42-70) years. Patients with ESBL-positive infections were significantly younger than those with ESBL-negative infections $(P=0.03)$. Most of the patients presented with acute illness (median [IQR] days of illness $~ 3[2-7]$ ) and there was no difference between the ESBL-positive and ESBL-negative groups in terms of clinical presentation.

Details of risk factors and outcomes are shown in Table 1. By univariable analysis, the presence of renal calculi (odds ratio [OR] 3.38, 95\% Cl: 1.20-9.48), chronic renal failure (OR 3.06, 95\% Cl: 1.14-8.24) and reported antibiotic use in the previous week (OR 4.40, 95\% Cl: 2.21-8.79) were significantly associated with ESBL positivity $(P<0.05$ for all). However, by multiple logistic regression, only reported antibiotic use in the previous week was independently significantly associated with ESBL positivity $(P<0.001$, OR 3.89 , 95\% Cl: 1.85-8.18).

Unfortunately, only 203 (56\%) of 360 patients had outcome data available; $144 / 203$ (71\%) were discharged alive, but the proportion of patients who died or were discharged moribund (a common outcome in Laos for cultural reasons) was significantly higher in the ESBL-positive group than in the ESBLnegative patients. However, patient outcomes were not independently associated with ESBL positivity by multiple logistic regression $(P=0.21)$. In the univariate analysis, patients infected with ESBL-producing $K$. pneumoniae were significantly younger and less frequently reported a history of fever compared with those infected with ESBL-producing $E$. coli. There were no other significant differences between two pathogens in term of clinical presentations, co-morbidities, and outcomes (Table 2).

All ESBL-producing $E$. coli and $K$. pneumoniae in this study were susceptible to imipenem and/or meropenem (Table 3). The proportion susceptible to gentamicin declined sharply in 2011 but was relatively stable from 2012 to 2014 , whereas all isolates were sensitive to amikacin apart from a single isolate in 2014 from a patient with biliary sepsis. For other groups of antibiotics, susceptibility rates were relatively consistent, with no obvious trend during the study period: chloramphenicol was active against $47-68 \%$ of isolates, followed by ofloxacin and ciprofloxacin (33-57\% susceptible). Fewer than half of the isolates appeared susceptible in vitro to co-amoxiclav and fewer than $30 \%$ to ceftazidime and co-trimoxazole.

\section{DISCUSSION}

Since 2000 , bloodstream isolates of $E$. coli and K. pneumoniae have been routinely screened for ESBL production at Mahosot Hospital. Results are reported to clinicians in real time. Since the first ESBL-producing $E$. coli identified in 2004, there has been a steady increase in the proportion of bacteremia caused by ESBL$\mathrm{E}$ in Vientiane. We previously reported that $9 \%$ of $E$. coli causing bloodstream infection between 2004 and 2009 were ESBL producers. ${ }^{11}$ This retrospective study confirms that the overall proportion of Enterobacteriaceae causing bacteremia that produce ESBL further increased 3-fold from 2010 to 2014.

All ESBL-E should not be considered as a homogeneous group. ${ }^{14}$ The reasons for the differences are unclear, but imply distinct epidemiological differences between the two species. Extended-spectrum beta-lactamase-producing $E$. coli was significantly more common than ESBL-producing $K$. pneumoniae as a cause of bacteremia in our population (15.28\% versus $4.72 \%$, $P<0.001)$. Interestingly, our study demonstrated different chronological trends for ESBL-producing $E$. coli bacteremia, which 

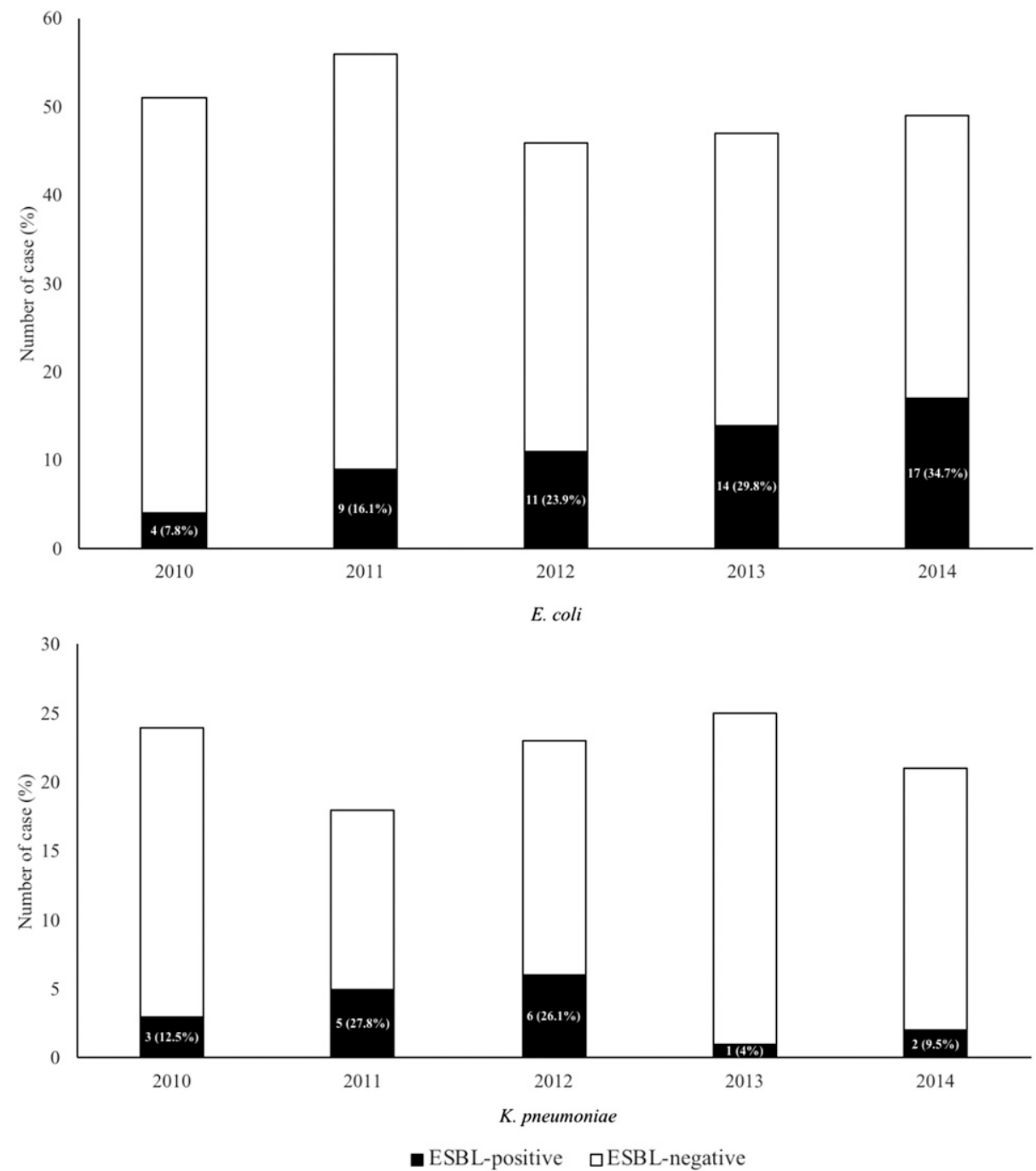

FIGURE 1. Extended-spectrum beta-lactamase (ESBL) production in Escherichia coli and Klebsiella pneumoniae blood culture isolates between 2010 and 2014 at Mahosot Hospital, Vientiane, Lao PDR.

increased in proportion nearly 5-fold during the study period, and ESBL-producing $K$. pneumoniae bacteremia, which initially doubled (from $12.5 \%$ in 2010 to $26.1 \%$ in 2012) but then declined to $4 \%$ and $9.5 \%$ in 2013 and 2014 , respectively. Similar observations have been made in other studies. ${ }^{15}$ We also observed that patients with ESBL-producing $K$. pneumoniae bacteremia were younger than those with ESBL-producing $E$. coli bacteremia, which has also been described by others. ${ }^{16}$

Overall, this increase in the frequency of bacteremia caused by ESBL-E has significant implications for the empirical management of community-acquired sepsis, which is usually treated with ceftriaxone in Laos. Moreover, most of these isolates were multiresistant, leading to severe problems in selecting appropriate agents to replace ceftriaxone. However, the picture is not entirely bleak, as the proportion of ESBL-E as causes of bacteremia is still lower in Laos than in some neighboring countries, which have been high for many years. ${ }^{7}$ The proportion of $E$. coli and $K$. pneumoniae causing community-acquired bacteremia that are ESBL positive varies between countries across South East Asia, ranging from $11.8 \%$ to $50 \%$ for $E$. coli and $11.4 \%$ to $43.8 \%$ for $K$. pneumoniae, respectively. ${ }^{17-20}$ For example, between 2004 and 2010, a retrospective, multicenter surveillance study in all provincial hospitals in northeast Thailand, which lies to the south and west of Laos across the Mekong river, found that ESBL was produced by $11.8 \%$ of $E$. coli and $11.4 \%$ of $K$. pneumoniae causing bacteremia, with an increasing year-on-year trend. ${ }^{17}$ In Cambodia between July 2007 and December 2010, $47.7 \%$ of $E$. coli and $43.8 \%$ of K. pneumoniae causing bacteremia produced ESBL. ${ }^{18} \mathrm{~A}$ retrospective study conducted at the National Hospital for Tropical Diseases in Hanoi, Vietnam, between January 2011 and December 2013 demonstrated that the proportions of $E$. coli and K. pneumoniae causing bacteremia that produced ESBL were $45 \%$ and $12.3 \%$, respectively. ${ }^{19}$ A more recent study conducted at three hospitals in Yangon, Myanmar, from July to December 2014 , found that $50 \%$ of $E$. coli and $43 \%$ of $K$. pneumoniae causing bacteremia produced ESBL. ${ }^{20}$ In a prospective multicenter study conducted in 28 tertiary hospitals across China from September 2013 to November 2014, the corresponding proportions were $55.5 \%$ and $16.7 \%$, respectively. ${ }^{21}$ The fact that Laos is still lagging behind these countries means that there may be a chance to intervene before the problem reaches the size of that in its neighbors. This should inform rapid policy decisions and implementation to prevent the situation worsening. 
TABLE 1

Characteristics and symptoms of patients with ESBL-negative and ESBL-positive Escherichia coli and Klebsiella pneumoniae bacteremia at the time of study admission (data shown as number (\%) unless indicated)

\begin{tabular}{|c|c|c|c|c|}
\hline & Total $(n=360)$ & ESBL negative $(n=288)$ & ESBL positive $(n=72)$ & $P$-value \\
\hline \multicolumn{5}{|l|}{ Demographic data } \\
\hline Age (years), median (IQR) & $57(42-70)$ & $58(43-70)$ & $53(32-65)$ & 0.03 \\
\hline$\leq 15$ years old, $n / N(\%)$ & $27 / 360(8)$ & $18 / 288(6)$ & $9 / 72(13)$ & 0.09 \\
\hline Male, $n / N(\%)$ & $143 / 360(40)$ & $108 / 288(38)$ & $35 / 72(49)$ & 0.09 \\
\hline \multicolumn{5}{|l|}{ Symptoms } \\
\hline Days ill, median (IQR) & $3(2-7)$ & $3(2-7)$ & $3.5(2-7)$ & 0.70 \\
\hline Fever, $n / N(\%)$ & $345 / 356(97)$ & $277 / 286(97)$ & $68 / 70(97)$ & 1.00 \\
\hline Rigors, $n / N(\%)$ & $230 / 354(65)$ & $190 / 285(67)$ & $40 / 69$ (58) & 0.17 \\
\hline Headache, $n / N(\%)$ & 197/355 (55) & $161 / 286(56)$ & 36/69 (52) & 0.50 \\
\hline Arthralgia, $n / N(\%)$ & $118 / 355(33)$ & 95/286 (33) & 23/69 (33) & 0.90 \\
\hline Back pain, $n / N(\%)$ & $123 / 355(35)$ & 99/286 (35) & $24 / 69$ (35) & 0.90 \\
\hline Myalgia, $n / N(\%)$ & $184 / 355(52)$ & $149 / 286(52)$ & $35 / 69$ (51) & 0.80 \\
\hline Retro-orbital pain, $n / N(\%)$ & $16 / 340(5)$ & $15 / 275(6)$ & $1 / 65(2)$ & 0.32 \\
\hline Jaundice, $n / N(\%)$ & 64/351 (18) & 51/284 (18) & 13/67 (19) & 0.70 \\
\hline Nausea, $n / N(\%)$ & $121 / 352(34)$ & 101/284 (36) & 20/68 (29) & 0.30 \\
\hline Vomiting, $n / N(\%)$ & 99/352 (28) & $82 / 284(28)$ & $17 / 68(25)$ & 0.50 \\
\hline Dysuria, $n / N(\%)$ & 62/352 (17) & 49/284 (17) & 13/68 (19) & 0.71 \\
\hline Diarrhea, $n / N(\%)$ & 67/351 (19) & $59 / 283(21)$ & 8/68 (12) & 0.08 \\
\hline Constipation, $n / N(\%)$ & $25 / 349(7)$ & $21 / 282(7)$ & $4 / 67(6)$ & 0.79 \\
\hline Abdominal pain, $n / N(\%)$ & $100 / 346(29)$ & $84 / 280(30)$ & 16/66 (24) & 0.30 \\
\hline Sore throat, $n / N(\%)$ & $14 / 346(4)$ & $11 / 280(4)$ & $3 / 66(5)$ & 0.78 \\
\hline Drowsiness, $n / N(\%)$ & $56 / 346(16)$ & 43/280 (15) & $13 / 66(20)$ & 0.38 \\
\hline \multicolumn{5}{|l|}{ Risk factors, $n / N(\%)$} \\
\hline Diabetes, $n / N(\%)$ & 88/335 (26) & $76 / 272(28)$ & 12/63 (19) & 0.31 \\
\hline Excessive alcohol use, $n / N(\%)$ & $34 / 317(11)$ & $27 / 257(11)$ & $7 / 60$ (12) & 0.79 \\
\hline Smoking, $n / N(\%)$ & 37/311 (12) & 28/253 (11) & $9 / 58(16)$ & 0.34 \\
\hline Chronic renal failure, $n / N(\%)$ & $18 / 341(5)$ & $11 / 278(4)$ & 7/64 (11) & 0.05 \\
\hline Renal calculi, $n / N(\%)$ & $20 / 336(6)$ & $12 / 275(4)$ & 8/61 (13) & 0.01 \\
\hline TB, $n / N(\%)$ & 6/236 (3) & $5 / 195(3)$ & $1 / 42(2)$ & 0.92 \\
\hline HIV, $n / N(\%)$ & $5 / 294(2)$ & $5 / 243(2)$ & $0 / 52(0)$ & 0.72 \\
\hline Steroid use, $n / N(\%)$ & 14/303 (5) & $11 / 252(4)$ & $3 / 52(6)$ & 0.80 \\
\hline $\begin{array}{l}\text { Reported antibiotic use in the previous } \\
\text { week, } n / N(\%)\end{array}$ & $61 / 235(26)$ & $38 / 192(20)$ & 23/44 (52) & $<0.001$ \\
\hline \multicolumn{5}{|l|}{ Outcome } \\
\hline Discharged alive, $n / N(\%)$ & $144 / 203(71)$ & $122 / 164(74)$ & 22/39 (56) & 0.04 \\
\hline Died in hospital, $n / N(\%)$ & 25/203 (12) & 16/164 (10) & 9/39 (23) & \\
\hline Discharged moribund, $n / N(\%)$ & $34 / 203(17)$ & $26 / 164(16)$ & $8 / 39(20)$ & \\
\hline
\end{tabular}

Our retrospective study found a number of factors that were associated with ESBL production in patients with $E$. coli and $K$. pneumoniae bacteremia, including renal calculi, chronic renal failure, and reported antibiotic use in the previous week. However, only reported antibiotic use in the previous week remained as a significant risk factor by multiple logistic regression. The recorded antibiotic use data did not, however, include the details of antibiotic class, length of, or reasons for prescription. Previous studies have also shown that healthcare-associated infection, obstructive urinary tract disease, chronic kidney disease, cerebrovascular disease, heart failure, previous major surgery, malignancies, and previous use of antibiotics were associated with bloodstream infection caused by ESBL-producing $E$. coli and K. pneumoniae. ${ }^{21-26}$ Furthermore, prior use of several different classes of antimicrobials, including extended-spectrum cephalosporins, fluoroquinolones, aminoglycosides, co-trimoxazole, and carbapenems, has repeatedly been associated with ESBLproducing $E$. coli and $K$. pneumoniae bacteremia, ${ }^{23,24,27-32}$ and a study from Spain showed that receipt of more than two different antibiotic classes in the preceding 90 days was the only predictor of $\mathrm{ESBL}$ production in patients with $E$. coli or K. pneumoniae bacteremia (OR 2.29, 95\% Cl: 1.35-3.88). ${ }^{33}$ Whether the link observed in our study was causal or was simply a reflection of the fact that blood cultures are more likely to be positive in patients on antibiotics who are infected with multiresistant organisms than with susceptible organisms is impossible to say. However, inappropriate antibiotic prescribing is common in Laos and there is a clear need to educate local clinicians about diagnostic stewardship and the prudent use of antibiotics. $^{34}$

Several studies have demonstrated that the mortality rate associated with ESBL-producing $E$. coli and $K$. pneumoniae bacteremia is significantly $(30-50 \%)$ higher than that of nonESBL-producing organisms, ${ }^{35-38}$ as it was in our study and associated with longer hospital stays and costs. ${ }^{39}$ The selection of appropriate antimicrobial therapy is an essential intervention to improve patient outcomes. ${ }^{40}$ This is particularly important during empirical treatment, although susceptibility results are still pending or in patients who are critically ill and are not responding to initial treatment with agents such as thirdgeneration cephalosporins. This study has shown that local ESBL-producing $E$. coli and $K$. pneumoniae causing bacteremia in Vientiane have a high rate of co-resistance to other groups of antibiotics but remain susceptible to meropenem and mostly to amikacin. This is consistent with previous studies from other centers. ${ }^{41-43}$ The suggested empirical treatment for patients with suspected sepsis in Mahosot Hospital is currently ceftriaxone, ${ }^{44}$ but this study raises the question as to whether this 
TABLE 2

Comparison of characteristics and symptoms of patients with ESBL-positive Escherichia coli and Klebsiella pneumoniae bacteremia (data shown as number (\%) unless indicated)

\begin{tabular}{|c|c|c|c|c|}
\hline & Total $(n=72)$ & ESBL-E. coli $(n=55)$ & ESBL-K. pneumoniae $(n=17)$ & $P$-value \\
\hline \multicolumn{5}{|l|}{ Demographic data } \\
\hline Age (years), median (IQR) & $53(32-65)$ & $56(45-65)$ & $24(0.41-54)$ & 0.0097 \\
\hline$\leq 15$ year old, $n / N(\%)$ & 9/72 (12) & 2/55 (3.6) & 7/17 (41) & $<0.001$ \\
\hline Male, $n / N(\%)$ & $35 / 72$ (49) & $26 / 55(47)$ & 9/17 (53) & 0.68 \\
\hline \multicolumn{5}{|l|}{ Symptoms } \\
\hline Days ill, median (IQR) & $3.5(2-7)$ & $3(2-7)$ & $4(2-7)$ & 0.77 \\
\hline Fever, $n / N(\%)$ & $68 / 70(97)$ & $55 / 55(100)$ & $13 / 15(86)$ & 0.04 \\
\hline Rigors, $n / N(\%)$ & 40/69 (58) & $34 / 54(63)$ & $6 / 15(40)$ & 0.11 \\
\hline Headache, $n / N(\%)$ & $36 / 69$ (52) & $31 / 54(57)$ & 5/15 (33) & 0.09 \\
\hline Arthralgia, $n / N(\%)$ & 23/69 (33) & 19/54 (35) & 4/15 (27) & 0.75 \\
\hline Back pain, $n / N(\%)$ & $24 / 69$ (35) & 18/54 (33) & $6 / 15(40)$ & 0.63 \\
\hline Myalgia, $n / N(\%)$ & $35 / 69$ (51) & 30/54 (55) & 5/15 (33) & 0.12 \\
\hline Retro-orbital pain, $n / N(\%)$ & $1 / 65(2)$ & $1 / 50(2)$ & $0 / 15(0)$ & 1.00 \\
\hline Jaundice, $n / N(\%)$ & 13/67 (19) & $12 / 52(23)$ & $1 / 15(7)$ & 0.26 \\
\hline Nausea, $n / N(\%)$ & $20 / 68$ (29) & $17 / 53(32)$ & $3 / 15(20)$ & 0.52 \\
\hline Vomiting, $n / N(\%)$ & $17 / 68(25)$ & $14 / 53(26)$ & $3 / 15(20)$ & 0.74 \\
\hline Dysuria, $n / N(\%)$ & $13 / 68(19)$ & $11 / 53(21)$ & $2 / 15(13)$ & 0.71 \\
\hline Diarrhea, $n / N(\%)$ & 8/68 (12) & 6/53 (11) & 2/15 (13) & 1.00 \\
\hline Constipation, $n / N(\%)$ & $4 / 67(6)$ & $4 / 53(7)$ & $0 / 14(0)$ & 0.57 \\
\hline Abdominal pain, $n / N^{\prime}(\%)$ & $16 / 66(24)$ & $12 / 51(23)$ & $4 / 15(27)$ & 1.00 \\
\hline Sore throat, $n / N(\%)$ & $3 / 66(5)$ & $2 / 51(4)$ & $1 / 15(7)$ & 0.54 \\
\hline Drowsiness, $n / N(\%)$ & $13 / 66(20)$ & $10 / 51(19)$ & $3 / 15(20)$ & 1.00 \\
\hline \multicolumn{5}{|l|}{ Risk factor } \\
\hline Diabetes, $n / N(\%)$ & 12/63 (19) & 9/51 (17) & 3/12 (25) & 0.25 \\
\hline Excess alcohol, $n / N(\%)$ & 7/60 (12) & 6/48 (12) & $1 / 12(8)$ & 1.00 \\
\hline Smokes, $n / N(\%)$ & $9 / 58(15)$ & $8 / 46(17)$ & $1 / 12(8)$ & 0.66 \\
\hline Chronic renal failure, $n / N(\%)$ & $7 / 64(11)$ & 6/50 (12) & $1 / 14(7)$ & 0.91 \\
\hline Renal calculi, $n / N(\%)$ & 8/61 (13) & 7/47 (15) & $1 / 14(7)$ & 0.63 \\
\hline Steroid use, $n / N(\%)$ & $3 / 52(6)$ & $2 / 41(5)$ & $1 / 11(9)$ & 0.75 \\
\hline $\begin{array}{l}\text { Reported antibiotic use in the previous } \\
\text { week, } n / N(\%)\end{array}$ & 23/44 (52) & $18 / 35(51)$ & $5 / 9(56)$ & 1.00 \\
\hline \multicolumn{5}{|l|}{ Outcome } \\
\hline Discharged alive, $n / N(\%)$ & $22 / 39(56)$ & $16 / 28(57)$ & $6 / 11(55)$ & 0.79 \\
\hline Died in hospital, $n / N(\%)$ & $9 / 39(23)$ & $7 / 28(25)$ & 2/11 (18) & \\
\hline Discharged moribund, $n / N(\%)$ & $8 / 39(20)$ & $5 / 28(18)$ & $3 / 11(27)$ & \\
\hline
\end{tabular}

$\mathrm{ESBL}=$ extended-spectrum beta-lactamase.

should be changed. However, carbapenems are expensive and not readily available in Laos and amikacin, although often used when indicated, is potentially toxic, especially as therapeutic drug monitoring is not locally available. There is also a risk of the emergence of resistance if these agents were more widely used. $^{45-47}$ Carbapenemase screening is already in place in Mahosot Hospital. We are currently investigating the use of rapid methods to detect ESBL production in patients with positive blood cultures growing Gram-negative bacilli in the hope that more targeted use of these valuable agents may be possible.

TABLE 3

Antimicrobial susceptibility of extended-spectrum beta-lactamase-producing Escherichia coli and Klebsiella pneumoniae isolated from blood cultures between 2010 and 2014 at Mahosot Hospital, Vientiane, Lao PDR

\begin{tabular}{|c|c|c|c|c|c|c|}
\hline Year & 2010 & 2011 & 2012 & 2013 & 2014 & \\
\hline Antimicrobial & $n / N(\%)$ & $n / N(\%)$ & $n / N(\%)$ & $n / N(\%)$ & $n / N(\%)$ & $P$-value \\
\hline Ampicillin & $0 / 7(0)$ & $0 / 14(0)$ & $0 / 17(0)$ & $0 / 15(0)$ & 0/19 (0) & NA \\
\hline Co-amoxiclav & 2/7 (29) & $3 / 14(21)$ & $8 / 17(47)$ & $7 / 15(47)$ & $4 / 19(21)$ & 0.55 \\
\hline Cefalotin & $0 / 7(0)$ & $0 / 14(0)$ & $0 / 17(0)$ & $0 / 15(0)$ & $0 / 19(0)$ & NA \\
\hline Ceftazidime & $1 / 5(20)$ & $3 / 12(25)$ & 4/14 (28) & $2 / 15(13)$ & 4/19 (21) & 0.96 \\
\hline Ceftriaxone & $0 / 7(0)$ & $0 / 14(0)$ & $0 / 17(0)$ & $0 / 15(0)$ & $0 / 19(0)$ & NA \\
\hline Ciprofloxacin & $1 / 1(100)$ & ND & ND & 6/13 (46) & $5 / 15$ (33) & 0.81 \\
\hline Chloramphenicol & $4 / 7(57)$ & $7 / 14(50)$ & $8 / 17(47)$ & $9 / 15(60)$ & $13 / 19(68)$ & 0.51 \\
\hline Doxycycline & ND & ND & ND & 1/4 (25) & 1/3 (33) & 1.00 \\
\hline Gentamicin & $5 / 7(71)$ & $3 / 14(21)$ & $8 / 17(47)$ & $8 / 15$ (53) & $10 / 19$ (52) & 0.15 \\
\hline Meropenem & $6 / 6(100)$ & 12/12 (100) & $15 / 15(100)$ & 15/15 (100) & $17 / 17(100)$ & NA \\
\hline Imipenem & 7/7 (100) & 13/13 (100) & 13/13 (100) & 15/15 (100) & $15 / 15(100)$ & NA \\
\hline Ofloxacin & $4 / 7(57)$ & $5 / 14(36)$ & $9 / 17(53)$ & $8 / 15(53)$ & $6 / 19(31)$ & 0.53 \\
\hline Co-trimoxazole & $0 / 7(0)$ & 3/14 (21) & 4/17 (23) & $4 / 15(27)$ & $3 / 19$ (16) & 0.70 \\
\hline Tetracycline & ND & $1 / 1(100)$ & ND & $3 / 11(27)$ & 2/11 (18) & 0.37 \\
\hline Amikacin & $6 / 6(100)$ & 12/12 (100) & 15/15 (100) & 15/15 (100) & 16/17 (94) & 1.00 \\
\hline
\end{tabular}

denominators reflect the fact that not all isolates were tested against each agent. 
Our study has several limitations. Because of its retrospective nature, some hospital charts could not be retrieved, and so it was not possible always to capture data about risk factors, comorbidities, and clinical outcomes related to ESBL$\mathrm{E}$ bacteremia. Second, this study was carried out in a single center and might not be applicable to other settings across Laos. Third, the estimated number of presumed hospitalacquired bacteremias is small and possibly exaggerated (maximum 10\% overall) and we have, therefore, not attempted to analyze the data by whether episodes were hospital or community acquired. Furthermore, blood cultures are not always collected on admission. Reported antibiotic use data are also limited in scope, particularly timings and class. Last, the data relate to 2010-2014 and because the epidemiology of AMR is likely to be changing constantly, expanded surveillance is essential.

\section{CONCLUSION}

Our study has demonstrated an alarming increase in the incidence of ESBL-producing $E$. coli and, to a lesser extent K. pneumoniae, bacteremia in Laos. Because most isolates were multiresistant, the effectiveness of antibiotics that are commonly used in the local setting is compromised. This emphasizes the need for appropriate local antibiotic guidelines based on accurate knowledge of local resistance patterns to improve patient outcomes and the importance of efforts to promote antimicrobial stewardship in Laos before the levels of AMR reach those of neighboring countries.

In addition, more detailed and ongoing AMR surveillance and research in the Lao PDR are also required, to build on this limited study.

Received April 21, 2019. Accepted for publication January 26, 2020. Published online March 9, 2020.

Acknowledgments: We are grateful to the staff at the Ministry of Health, the University of Health Sciences, and the Directors of Mahosot Hospital for their support of this work. We thank the staff of the Microbiology Laboratory, Mahosot Hospital, who undertook the routine bacteriological testing during the period of the study and the clinical staff of Mahosot Hospital who submitted the specimens and clinical data.

Financial support: This work was supported by the Wellcome Trust, United Kingdom, through funding for the Lao-Oxford-Mahosot Hospital-Wellcome Trust Research Unit.

Disclosure: Ethical approval for the study was granted by the Oxford Tropical Research Ethics Committee and the Lao National Ethics Committee for Health Research.

Authors' addresses: Ko Chang and Valy Keoluangkhot, Adult Infectious Diseases Ward, Mahosot Hospital, Vientiane, Laos, E-mails: me.chang2009@gmail.com and valy.keoluangkhot@gmail.com. Sayaphet Rattanavong, Viengmon Davong, Manivanh Vongsouvath, and Manophab Luangraj, Lao-Oxford-Mahosot Hospital-Wellcome Trust Research Unit (LOMWRU), Microbiology Laboratory, Mahosot Hospital, Vientiane, Laos, E-mails: sayaphet@tropmedres.ac, viengmon@ tropmedres.ac, manivanh@tropmedres.ac, and manophab.|@tropmedres. ac. Mayfong Mayxay, Lao-Oxford-Mahosot Hospital-Wellcome Trust Research Unit (LOMWRU), Microbiology Laboratory, Mahosot Hospital, Vientiane, Laos, Centre for Tropical Medicine and Global Health, University of Oxford, Oxford, United Kingdom, and Institute of Research and Education Development (IRED), University of Health Sciences, Vientiane, Laos, E-mail: mayfong@tropmedres. ac. Andrew J. H. Simpson, Lao-Oxford-Mahosot Hospital-Wellcome Trust Research Unit (LOMWRU), Microbiology Laboratory, Mahosot Hospital, Vientiane, Laos, and Centre for Tropical Medicine and
Global Health, University of Oxford, Oxford, United Kingdom, E-mail: andrew.s@tropmedres.ac. Paul N. Newton and David A. B. Dance, Lao-Oxford-Mahosot Hospital-Wellcome Trust Research Unit (LOMWRU), Microbiology Laboratory, Mahosot Hospital, Vientiane, Laos, Centre for Tropical Medicine and Global Health, University of Oxford, Oxford, United Kingdom, and Faculty of Infectious and Tropical Diseases, London School of Hygiene and Tropical Medicine, London, United Kingdom, E-mails: paul.newton@tropmedres.ac and david.d@ tropmedres.ac.

This is an open-access article distributed under the terms of the Creative Commons Attribution (CC-BY) License, which permits unrestricted use, distribution, and reproduction in any medium, provided the original author and source are credited.

\section{REFERENCES}

1. Laupland KB, Church DL, 2014. Population-based epidemiology and microbiology of community-onset bloodstream infections. Clin Microbiol Rev 27: 647-664.

2. Laupland KB, 2013. Incidence of bloodstream infection: a review of population-based studies. Clin Microbiol Infect 19: 492-500.

3. de Kraker ME, Davey PG, Grundmann H, 2011. Mortality and hospital stay associated with resistant Staphylococcus aureus and Escherichia coli bacteremia: estimating the burden of antibiotic resistance in Europe. PLoS Med 8: e1001104.

4. Lim C, Takahashi E, Hongsuwan M, Wuthiekanun V, Thamlikitkul V, Hinjoy S, Day NP, Peacock SJ, Limmathurotsakul D, 2016. Epidemiology and burden of multidrug-resistant bacterial infection in a developing country. Elife 5: e18082.

5. Paterson DL, 2006. Resistance in gram-negative bacteria: Enterobacteriaceae. Am J Infect Control 34 (Suppl 1): S20S28.

6. Tacconelli E et al., 2018. Discovery, research, and development of new antibiotics: the WHO priority list of antibiotic-resistant bacteria and tuberculosis. Lancet Infect Dis 18: 318-327.

7. Hawser SP, Bouchillon SK, Hoban DJ, Badal RE, Hsueh PR, Paterson DL, 2009. Emergence of high levels of extendedspectrum-beta-lactamase-producing gram-negative bacilli in the Asia-Pacific region: data from the study for monitoring antimicrobial resistance trends (SMART) program, 2007. Antimicrob Agents Chemother 53: 3280-3284.

8. Suwantarat N, Carroll KC, 2016. Epidemiology and molecular characterization of multidrug-resistant gram-negative bacteria in southeast Asia. Antimicrob Resist Infect Control 5: 15.

9. Kiratisin P, Chongthaleong A, Tan TY, Lagamayo E, Roberts S, Garcia J, Davies T, 2012. Comparative in vitro activity of carbapenems against major gram-negative pathogens: results of Asia-Pacific surveillance from the COMPACT II study. Int $J$ Antimicrob Agents 39: 311-316.

10. Phetsouvanh $R$ et al., 2006. Causes of community-acquired bacteremia and patterns of antimicrobial resistance in Vientiane, Laos. Am J Trop Med Hyg 75: 978-985.

11. Stoesser N, Crook DW, Moore CE, Phetsouvanh R, Chansamouth V, Newton PN, Jones N, 2012. Characteristics of CTX-M ESBLproducing Escherichia coli isolates from the Lao People's Democratic Republic, 2004-09. J Antimicrob Chemother 67: 240-242.

12. Stoesser $\mathrm{N}$ et al., 2015. Colonization with Enterobacteriaceae producing ESBLs in children attending pre-school childcare facilities in the Lao People's Democratic Republic. J Antimicrob Chemother 70: 1893-1897.

13. CLSI, 2014. Performance Standards for Antimicrobial Susceptibility Testing: Twenty-Fourth Informational Supplement. CLSI Document M100-S24. Wayne, PA: Clinical and Laboratory Standards Institute.

14. Scheuerman $O$ et al., 2018. Comparison of predictors and mortality between bloodstream infections caused by ESBLproducing Escherichia coli and ESBL-producing Klebsiella pneumoniae. Infect Control Hosp Epidemiol 39: 660-667.

15. Romero L, Lopez L, Rodriguez-Bano J, Ramon Hernandez J, Martinez-Martinez L, Pascual A, 2005. Long-term study of the frequency of Escherichia coli and Klebsiella pneumoniae isolates producing extended-spectrum beta-lactamases. Clin Microbiol Infect 11: 625-631. 
16. Sakellariou C, Gürntke S, Steinmetz I, Kohler C, Pfeifer $Y$, Gastmeier P, Schwab F, Kola A, Deja M, Leistner R, 2016. Sepsis caused by extended-spectrum beta-lactamase (ESBL)positive $K$. pneumoniae and E. coli: comparison of severity of sepsis, delay of anti-infective therapy and ESBL genotype. PLoS One 11: e0158039.

17. Kanoksil M, Jatapai A, Peacock SJ, Limmathurotsakul D, 2013. Epidemiology, microbiology and mortality associated with community-acquired bacteremia in northeast Thailand: a multicenter surveillance study. PLoS One 8: e54714.

18. Vlieghe ER, Phe T, De Smet B, Veng HC, Kham C, Lim K, Koole O, Lynen L, Peetermans WE, Jacobs JA, 2013. Bloodstream infection among adults in Phnom Penh, Cambodia: key pathogens and resistance patterns. PLoS One 8: e59775.

19. Dat VQ et al., 2017. Bacterial bloodstream infections in a tertiary infectious diseases hospital in northern Vietnam: aetiology, drug resistance, and treatment outcome. BMC Infect Dis 17: 493.

20. Myat TO, Hannaway RF, Zin KN, Htike WW, Win KK, Crump JA, Murdoch DR, Ussher JE, 2017. ESBL- and carbapenemaseproducing Enterobacteriaceae in patients with bacteremia, Yangon, Myanmar, 2014. Emerg Infect Dis 23: 857-859.

21. Quan J, Zhao D, Liu L, Chen Y, Zhou J, Jiang Y, Du X, Zhou Z, Akova M, Yu Y, 2017. High prevalence of ESBL-producing Escherichia coli and Klebsiella pneumoniae in communityonset bloodstream infections in China. J Antimicrob Chemother 72: 273-280.

22. Wu U-I, Yang C-S, Chen W-C, Chen Y-C, Chang S-C, 2010. Risk factors for bloodstream infections due to extended-spectrum $\beta$-lactamase-producing Escherichia coli. J Microbiol Immunol Infect 43: 310-316.

23. Khrongwong M, Chatgaew C, Chaita S, 2011. Risk factors of extended-spectrum beta-lactamase producing Enterobacteriaceae bacteremia in Thai emergency department: a retrospective case-control study. Asian Biomed 5: 129-138.

24. Kim SH et al., 2013. Escherichia coli and Klebsiella pneumoniae bacteremia in patients with neutropenic fever: factors associated with extended-spectrum beta-lactamase production and its impact on outcome. Ann Hematol 92: 533-541.

25. Kumar M, Dutta R, Saxena S, Singhal S, 2015. Risk factor analysis in clinical isolates of ESBL and MBL (including NDM-1) producing Escherichia coli and Klebsiella species in a tertiary care hospital. J Clin Diagn Res 9: DC08-DC13.

26. Nakai H, Hagihara M, Kato H, Hirai J, Nishiyama N, Koizumi $Y$, Sakanashi D, Suematsu H, Yamagishi Y, Mikamo H, 2016. Prevalence and risk factors of infections caused by extendedspectrum beta-lactamase (ESBL)-producing Enterobacteriaceae. $J$ Infect Chemother 22: 319-326.

27. Lautenbach E, Patel JB, Bilker WB, Edelstein PH, Fishman NO, 2001. Extended-spectrum beta-lactamase-producing Escherichia coli and Klebsiella pneumoniae: risk factors for infection and impact of resistance on outcomes. Clin Infect Dis 32: 1162-1171.

28. Du B, Long Y, Liu H, Chen D, Liu D, Xu Y, Xie X, 2002. Extendedspectrum beta-lactamase-producing Escherichia coli and Klebsiella pneumoniae bloodstream infection: risk factors and clinical outcome. Intensive Care Med 28: 1718-1723.

29. Martinez JA, Aguilar J, Almela M, Marco F, Soriano A, Lopez F, Balasso V, Pozo L, Mensa J, 2006. Prior use of carbapenems may be a significant risk factor for extended-spectrum betalactamase-producing Escherichia coli or Klebsiella spp. in patients with bacteraemia. J Antimicrob Chemother 58: 1082-1085.

30. Chen YH, Yao WZ, Wu R, Zhou QT, Liu ZY, Zhang XW, 2008. Risk factors and prognosis in 31 patients with extended-spectrum beta-lactamase producing Escherichia coli and Klebsiella pneumoniae bloodstream infection. Zhonghua Jie He He Hu Xi Za Zhi 31: 815-819.

31. Rodríguez-Baño J, Navarro MD, Romero L, Muniain MA, de Cueto M, Gálvez J, Perea EJ, Pascual A, 2008. Risk-factors for emerging bloodstream infections caused by extended-spectrum $\beta$-lactamase-producing Escherichia coli. Clin Microbiol Infect 14: 180-183.

32. Razazi K, Derde LPG, Verachten M, Legrand P, Lesprit P, BrunBuisson C, 2012. Clinical impact and risk factors for colonization with extended-spectrum $\beta$-lactamase-producing bacteria in the intensive care unit. Intensive Care Med 38: 1769-1778.

33. Ferrández Quirante O, Grau Cerrato S, Luque Pardos S, 2011. Risk factors for bloodstream infections caused by extendedspectrum $\beta$-lactamase-producing Escherichia coli and Klebsiella pneumoniae. Braz J Infect Dis 15: 370-376.

34. Quet F et al., 2015. Antibiotic prescription behaviours in Lao People's Democratic Republic: a knowledge, attitude and practice survey. Bull World Health Org 93: 219-227.

35. Falcone $\mathrm{M}$ et al., 2014. Role of empirical and targeted therapy in hospitalized patients with bloodstream infections caused by ESBL-producing Enterobacteriaceae. Ann Ig 26: 293-304.

36. Chopra $T$ et al., 2015. Risk factors for bloodstream infection caused by extended-spectrum beta-lactamase-producing Escherichia coli and Klebsiella pneumoniae: a focus on antimicrobials including cefepime. Am J Infect Control 43: 719-723.

37. Superti SV, Augusti G, Zavascki AP, 2009. Risk factors for and mortality of extended-spectrum- $\beta$-lactamase-producing Klebsiella pneumoniae and Escherichia coli nosocomial bloodstream infections. Rev Inst Med Trop São Paulo 51: 211-216.

38. Rottier WC, Ammerlaan HS, Bonten MJ, 2012. Effects of confounders and intermediates on the association of bacteraemia caused by extended-spectrum beta-lactamase-producing Enterobacteriaceae and patient outcome: a meta-analysis. $J$ Antimicrob Chemother 67: 1311-1320.

39. Schwaber MJ, Navon-Venezia S, Kaye KS, Ben-Ami R, Schwartz $D$, Carmeli Y, 2006. Clinical and economic impact of bacteremia with extended-spectrum beta-lactamase-producing Enterobacteriaceae. Antimicrob Agents Chemother 50: 1257-1262.

40. Tumbarello $\mathrm{M}$ et al., 2007. Predictors of mortality in patients with bloodstream infections caused by extended-spectrum-betalactamase-producing Enterobacteriaceae: importance of inadequate initial antimicrobial treatment. Antimicrob Agents Chemother 51: 1987-1994.

41. Vlieghe ER, Huang TD, Phe T, Bogaerts P, Berhin C, De Smet B, Peetermans WE, Jacobs JA, Glupczynski Y, 2015. Prevalence and distribution of beta-lactamase coding genes in thirdgeneration cephalosporin-resistant Enterobacteriaceae from bloodstream infections in Cambodia. Eur J Clin Microbiol Infect Dis 34: 1223-1229.

42. Noguchi T, Matsumura Y, Yamamoto M, Nagao M, Takakura S, Ichiyama S, 2017. Clinical and microbiologic characteristics of cefotaxime-non-susceptible Enterobacteriaceae bacteremia: a case control study. BMC Infect Dis 17: 44.

43. Sangare SA et al., 2016. Prevalence of ESBL-producing Enterobacteriaceae isolated from blood cultures in Mali. $J$ Infect Dev Ctries 10: 1059-1064.

44. 2013. Mahosost Microbiology Review 7: 15.

45. Kritsotakis El, Tsioutis C, Roumbelaki M, Christidou A, Gikas A, 2011. Antibiotic use and the risk of carbapenem-resistant extended-spectrum-\{beta\}-lactamase-producing Klebsiella pneumoniae infection in hospitalized patients: results of a double case-control study. J Antimicrob Chemother 66: 1383-1391.

46. Netikul T, Kiratisin P, 2015. Genetic characterization of carbapenem-resistant Enterobacteriaceae and the spread of carbapenem-resistant Klebsiella pneumoniae ST340 at a university hospital in Thailand. PLoS One 10: e0139116.

47. Munoz-Price LS et al., 2013. Clinical epidemiology of the global expansion of Klebsiella pneumoniae carbapenemases. Lancet Infect Dis 13: 785-796. 